\title{
Simple Synthesis of Functionalized Paramagnetic Beads for Nucleic Acid Purification and Manipulation
}

Phil Oberacker ${ }^{1,4, \text {, }, ~ P e t e r ~ S t e p p e r ~}{ }^{1, ~ \#, ~ D o n n a ~ B o n d ~}{ }^{2}$, Katharina Hipp ${ }^{3}$, Timothy A. Hore ${ }^{2, *}$ and Tomasz P. Jurkowski, *

\author{
${ }^{1}$ Department of Biochemistry, Institute of Biochemistry and Technical Biochemistry, University of \\ Stuttgart, Stuttgart, Germany; ${ }^{2}$ Department of Anatomy, University of Otago, Dunedin, New Zealand; \\ ${ }^{3}$ Max Planck Institute for Developmental Biology, Tübingen, Germany; ${ }^{4}$ School of Biosciences, \\ Cardiff University, Cardiff, Wales, United Kingdom \\ *For correspondence: jurkowskit@cardiff.ac.uk; tim.hore@otago.ac.nz \\ \#Contributed equally to this work
}

\begin{abstract}
[Abstract] The purification of nucleic acids is one of the most common procedures employed in modern molecular biology laboratories. Typically, commercial column-based protocols are utilized to isolate DNA or RNA from various sources. However, these methods not only require specialized equipment, but are also extremely expensive for high-throughput applications. Although an elegant answer to this issue can be provided by paramagnetic beads, bead-based open-source protocols have been limited in the past. Here, we provide an easy to follow step-by-step manual for the synthesis of paramagnetic beads, as well as their functionalization with either a silica- or a carboxyl-surface that can be used to replace the commercial columns with self-made magnetic beads. Together with a variety of detailed protocols for their use in high-throughput nucleic acids extractions, this bead synthesis method forms the recently published open platform Bio-On-Magnetic-Beads (BOMB), which is available on PLOS Biology (Oberacker et al., 2019). Updated protocols can be found on the associated webpage (https://bomb.bio).
\end{abstract}

Keywords: Carboxyl-coating, $\mathrm{Fe}_{3} \mathrm{O}_{4}$, Magnetic beads, Magnetic nanoparticles, Magnetic separation, Bead synthesis, Methacrylic acid, Silica-coating, $\mathrm{SiO}_{2}$, Surface functionalization

[Background] The ability to achieve solid-phase reversible immobilization (SPRI) (DeAngelis et al., 1995 ) is one of the most useful characteristics of functionalized paramagnetic nanoparticles (MNPs). Together with their scalability and potential for automation, MNPs are ideal candidates for the development of high-throughput protocols regarding nucleic acid purification (Hawkins et al., 1994; DeAngelis et al., 1995; Rohland and Reich, 2012). The small nano- or microparticles are able to reversibly bind nucleic acids under dehydrating conditions and can be safely immobilized by a strong magnet during consecutive wash or manipulation steps. Bead-based protocols can be adapted easily for multi-well formats, allowing the processing of hundreds of samples simultaneously. Additionally, MNPs are very cost-effective as the beads can be obtained very cheaply, both commercially and selfmade. Although their advantages over more established column-based methods are obvious, 
surprisingly little effort has been put in the development of open-source protocols featuring paramagnetic beads.

Protocols for the synthesis of ferrite nanoparticles have been published before (reviewed in Houshiar et al., 2014; Singh et al., 2014; Wu et al., 2015; Majidi et al., 2016). We adopted the commonly used co-precipitation method due to its efficient and robust performance and the fact that it does not require any specialized equipment (Choi et al., 2007). For this, a solution of $\mathrm{FeCl}_{2}$ and $\mathrm{FeCl}_{3}$ in a 1:2 molar ratio is prepared and slowly dripped into a preheated $\mathrm{NaOH}$ solution. This forms a black precipitate consisting of $\mathrm{Fe}_{3} \mathrm{O}_{4}$ particles with an approximate diameter of 5 to $20 \mathrm{~nm}$ as judged by electron microscopy (Figure 1A). As oxygen is known to interfere with ferrite precipitation reactions, the $\mathrm{NaOH}$ solution should be degassed and/or preheated to $80^{\circ} \mathrm{C}$ or higher before adding the iron solution. After the synthesis reaction, the nanoparticles are washed multiple times with deionized water. We recommend starting the respective coating reaction immediately after the synthesis as the particles are prone to oxidation. However, it is possible to stabilize them using various chemicals, such as detergents, sodium oleate or polyvinylpyrrolidone (PVP) (reviewed in Singh et al., 2014; Majidi et al., 2016). Upon lyophilization, the core MNPs can be stored in an air-tight container under inert atmosphere.

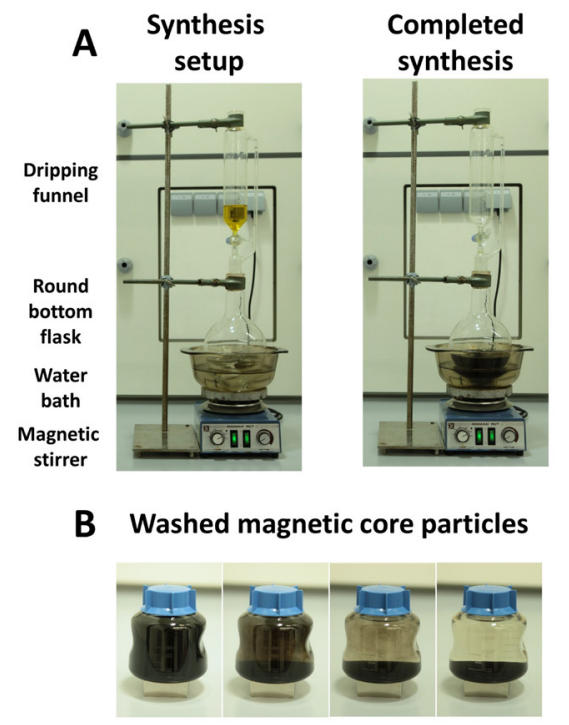

C Magnetic core particles


D Silica-coated beads
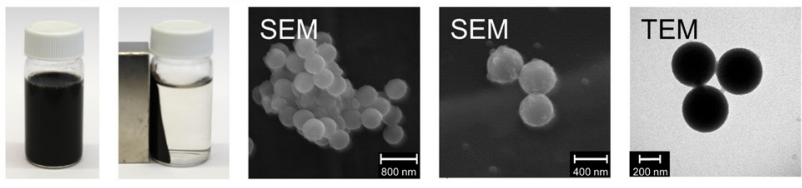

E Carboxyl-coated beads

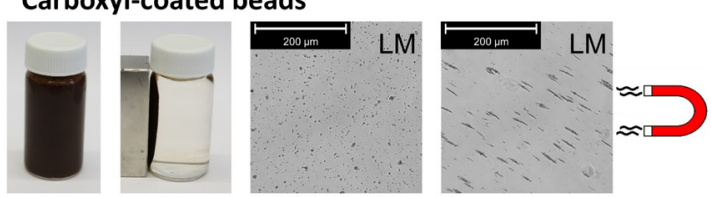

Figure 1. Synthesis of BOMB paramagnetic beads. A. Equipment setup for BOMB MNP synthesis. Place a magnetic stir bar in both the water bath as well as the round bottom flask in order to avoid heat accumulation. B. Synthesized and washed paramagnetic core particles. C. Paramagnetic core particles in transmission electron microscopy (TEM). D. Silica-coated paramagnetic beads in scanning electron microscopy (SEM) and TEM. E. Carboxyl-coated paramagnetic beads in light microscopy (LM), with and without an applied magnet.

Encasing of the ferrite nanoparticles in a silica coat prevents oxidation and leakage of iron ions and provides an inert surface for nucleic acid precipitation without the risk of irreversible binding. The silica deposition reaction outlined here is based on a modified version of the Stöber method (Stöber et al., 
1968). It utilizes tetraethyl orthosilicate (TEOS) which is hydrolyzed in a basic environment, leading to the deposition of a $\mathrm{SiO}_{2}$ layer surrounding the paramagnetic core. Conveniently, the thickness of the silica coat, and therefore the size of the beads, can be controlled by the amount of TEOS added to the reaction (Kim et al., 2007), allowing a precise adjustment depending on the respective downstream application. The provided standard coating protocol yields silica-coated beads with an average diameter of approximately $400 \mathrm{~nm}$ (Figure 1B), which perform well for a wide range of nucleic acid purification and manipulation experiments from bacteria, mammalian cells, tissues (Figure 2 ) and many more (Oberacker et al., 2019).

A

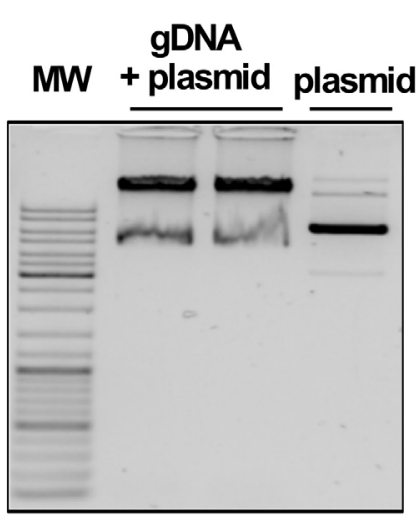

B

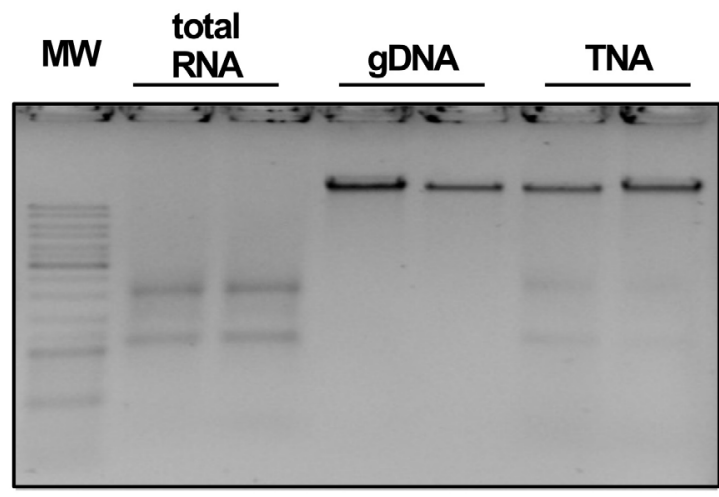

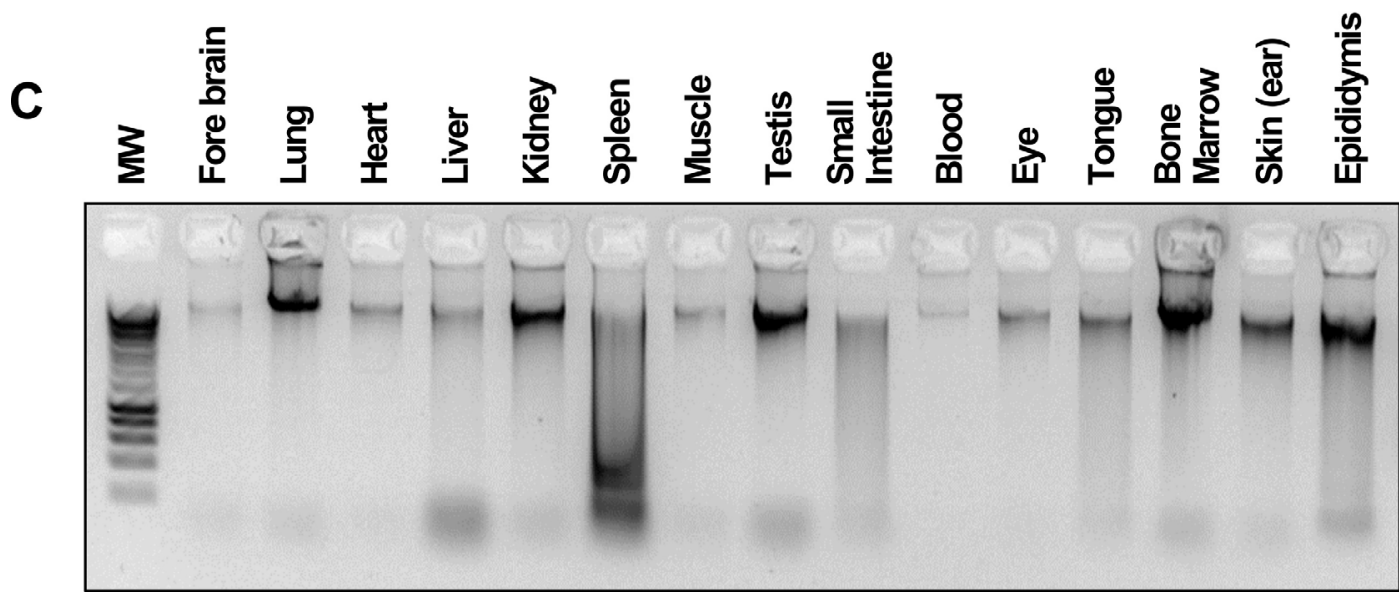

Figure 2. BOMB nucleic acid extractions. Data and figures are taken from Oberacker et al. (2019). A. Total DNA and plasmid extracted from E. coil. MW: GeneRuler DNA Ladder Mix (Thermo). B. TNA, DNA and RNA isolation from HEK293 cells. MW: GeneRuler DNA Ladder Mix (Thermo). C. gDNA isolated from various rabbit (O. cuniculus) tissues. MW: Hyperladder I (Bioline).

As an alternative to a silica-coat around the ferrite particles, a layer of a carboxyl-modified polymer can provide stability as well as a surface with a weak negative charge, thus altering the electrostatic interaction with nucleic acids and consequently the functionality of the beads (Figure 1C). Here, we use methacrylic acid (MAA) monomers, which form a layer of polymethacrylic acid (PMAA) around the ferrite core particles during a free-radical retrograde precipitation polymerization reaction (Aggarwal et 
al., 1996). Potentially, carboxyl- and silica-surfaces can be further modified to enable a broad range of additional functionalities to the paramagnetic beads.

\section{Materials and Reagents}

1. $60 \mathrm{ml}$ syringe (HENKE SASS WOLF, catalog number: 8300006680 )

2. $0.2 \mu \mathrm{m}$ sterile filter (Sarstedt, catalog number: 83.1826.001)

3. Protective clothing (lab coat, eye protection, gloves)

4. Stand with boss head sockets and retort clamps (Bochem, catalog numbers: 5000, 5300, 5401)

5. $250 \mathrm{ml}$ dripping funnel (Buhler, NS29/32)

6. Heat resistant bowl for a water bath

7. Heat resistant $2.5 \mathrm{~L}$ bottle

8. 0.5 or $1 \mathrm{~L}$ round bottom flask (Schott Duran, NS 29/32, catalog number: 2172154)

9. Sterile plastic $0.5 \mathrm{~L}$ bottles (Corning ${ }^{\circledR}$ Costar $^{\circledR}$, Nunc ${ }^{\circledR} \mathrm{T} 175$ uncoated PS flask)

10. $50 \mathrm{ml}$ conical centrifuge tube (Sarstedt, catalog number: 62.547 .004 )

11. Ammonia solution ( $25 \% \mathrm{NH}_{4} \mathrm{OH}$ ) (EMD Millipore, catalog number: 1.05432$)$

12. Ethanol $\left(99 \% \mathrm{C}_{2} \mathrm{H}_{6} \mathrm{O}\right)$ (Riedel-de Haën, catalog number: 34963$)$ or lower grade

13. Hydrochloric acid ( $37 \% \mathrm{HCl}$ fuming) (Roth Chemicals, catalog number: 4625.1$)$

14. Iron(II) chloride 4-hydrate ( $\left.\geq 99 \% \mathrm{FeCl}_{2} \cdot 4 \mathrm{H}_{2} \mathrm{O}\right)$ (Honeywell/Fluka, catalog number: 44939)

15. Iron(III) chloride ( $\geq 98.5 \% \mathrm{FeCl}_{3}$ ) (Roth Chemicals, catalog number: 5192.3 )

16. Methacrylic acid ( $\geq 99 \% \mathrm{C}_{4} \mathrm{H}_{6} \mathrm{O}_{2}$ ) (Merck, catalog number: 155721)

17. Potassium persulfate ( $\geq 99 \% \mathrm{~K}_{2} \mathrm{~S}_{2} \mathrm{O}_{8}$ ) (Merck, catalog number: 216224 )

18. Sodium dodecyl sulfate $\left(\mathrm{NaC}_{12} \mathrm{H}_{25} \mathrm{SO}_{4}\right)$ (Merck, catalog number: L3771-100G)

19. Sodium hydroxide ( $\geq 99 \% \mathrm{NaOH}$ ) (Roth Chemicals, catalog number: 6771.1 )

20. Tetraethyl orthosilicate $\left(\geq 99 \% \mathrm{Si}\left(\mathrm{OC}_{2} \mathrm{H}_{5}\right)_{4}\right)$ (Merck, catalog number: 86578)

21. $2 \mathrm{M} \mathrm{NaOH}$ (see Recipes)

22. $0.1 \mathrm{M} \mathrm{HCl}$ (see Recipes)

23. $\mathrm{Fe} 2 / 3$ solution (see Recipes)

Note: Please consult appropriate MSDS information before working with these chemicals! Use a lab coat, gloves and eye protection at all times! The chemicals are available from other providers as well. No preference is given to the indicated vendors.

\section{Equipment}

1. Fume hood

2. Vacuum filtration systems (Corning, catalog number: UD-29530-02)

3. Magnetic stirrer with heating (Heidolph, catalog number: 504-10000-00)

4. Magnetic stir bars 
5. Strong neodymium permanent magnet (e.g., Neodymium disc magnet $80 \times 10 \mathrm{~mm}$ grade $\mathrm{N} 45$ adhesive force $\sim 300 \mathrm{~kg}$-be extremely careful when using such strong magnets!)

\section{Procedure}

All of the following written protocols are also provided as a flowchart (Figure 3 ).

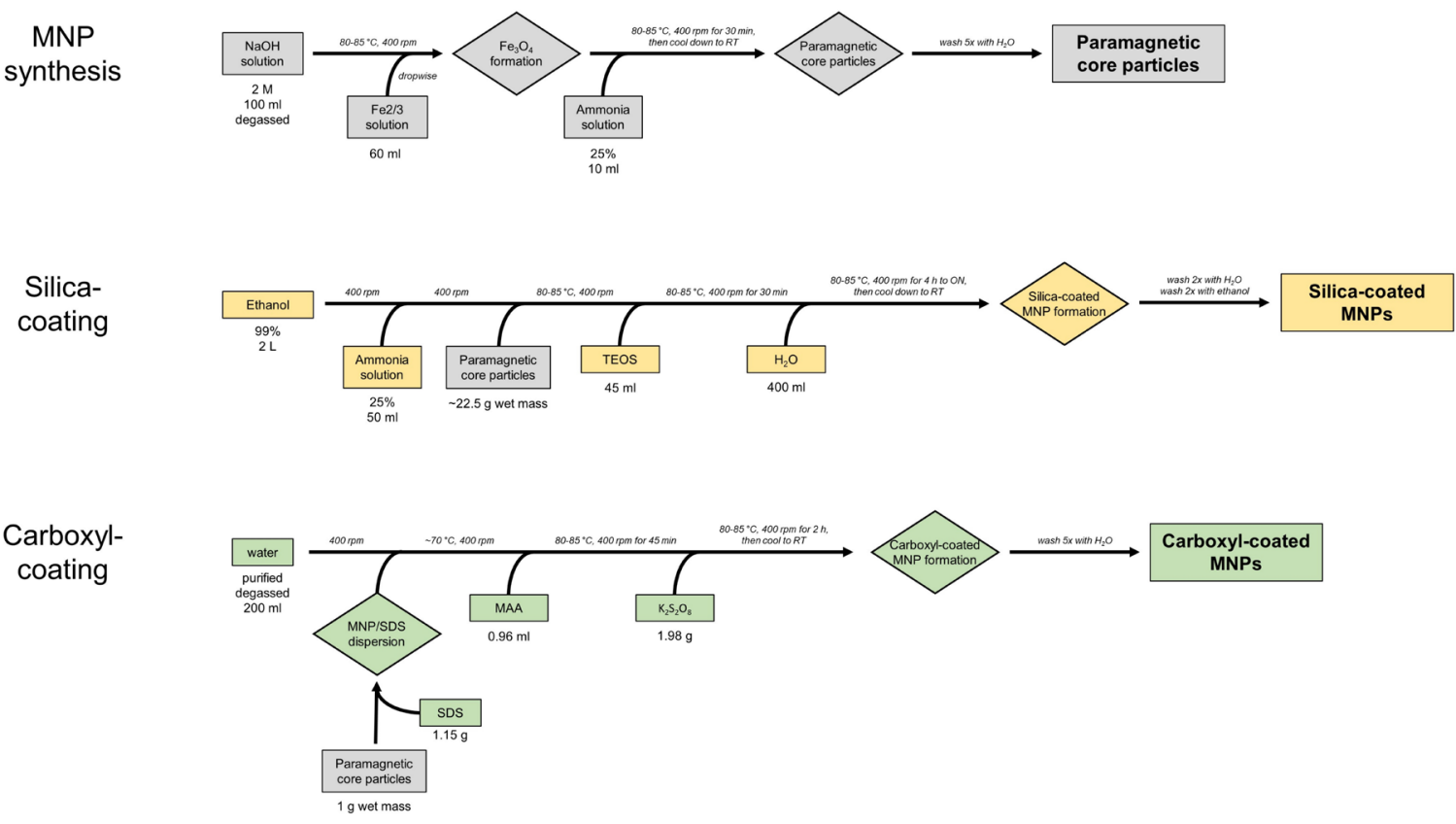

Figure 3. BOMB MNP protocols. Protocol workflow for synthesis of MNP core particles (grey) and their coating with either silica (yellow) or carboxyl (green).

A. BOMB synthesis of MNPs

Note: All procedures can be performed under inert argon atmosphere (alternatively purging the system with high purity $\mathrm{N}_{2}$ for 30 min should be sufficient. We generally perform the reaction at atmospheric oxygen levels, however degas and heat up the solutions before use).

1. Degas the $\mathrm{NaOH}$ solution by filtering it with a vacuum filtering system-keep the vacuum running for $1 \mathrm{~h}$ while magnetically stirred.

2. Assemble the round bottom flask, dripping funnel and water bath according to Figure $1 \mathrm{~A}$ and place on a magnetic stirrer. Add $100 \mathrm{ml}$ of degassed $2 \mathrm{M} \mathrm{NaOH}$ to the collection flask on the bottom and the $\mathrm{Fe} 2 / 3$ solution to the dripping funnel. Heat up the flask containing the $2 \mathrm{M} \mathrm{NaOH}$ solution to $80-85^{\circ} \mathrm{C}$.

Note: Using freshly made Fe2/3 solution with the correct iron salts is crucial for the success of the reaction. Otherwise, the forming iron precipitate might lead to lower stability of MNPs as can be observed during washing steps or storing the beads.

3. Add $60 \mathrm{ml} \mathrm{Fe} 2 / 3$ solution drop wise into $100 \mathrm{ml}$ of preheated $\left(80-85^{\circ} \mathrm{C}\right) 2 \mathrm{M} \mathrm{NaOH}$ solution 
while stirring vigorously (> $400 \mathrm{rpm})$.

Note: $A$ black precipitate of $\mathrm{Fe}_{3} \mathrm{O}_{4}\left(\mathrm{FeO} \cdot \mathrm{Fe}_{2} \mathrm{O}_{3}\right)$ is formed.

4. After the complete $\mathrm{Fe} 2 / 3$ solution was added, add $10 \mathrm{ml}$ of $25 \%$ ammonia solution to the reaction mixture. Stir for another $30 \mathrm{~min}$ and then cool to RT.

5. Transfer the solution to a sterile plastic container and magnetically pellet the black precipitate (MNPs), discard the supernatant.

Note: Ammonia containing trash needs to be discarded appropriately.

6. Resuspend in $200 \mathrm{ml}$ of $\mathrm{ddH}_{2} \mathrm{O}$, pellet magnetically and discard supernatant. Repeat for a total of 5 washes (or until the $\mathrm{pH}$ of the wash reaches $\sim 7$ ).

7. After the last wash, resuspend the MNPs in $30-40 \mathrm{ml}$ of $\mathrm{ddH}_{2} \mathrm{O}$ and transfer to a fresh $50 \mathrm{ml}$ tube.

Note: To measure the synthesis yield, magnetically pellet the MNPs, remove the water and weigh the product wet-mass. Alternatively, flash freeze the pelleted MNPs in liquid nitrogen and lyophilize and measure the dry mass. Lyophilised MNPs in $\mathrm{N}_{2}$ or argon atmosphere can be stored in a closed container at RT for a longer time. The wet MNPs slowly oxidize over time; therefore, we recommend coating them within a week.

\section{B. BOMB Silica-coating}

Note: All procedures can be performed under inert $\mathrm{N}_{2}$ atmosphere or atmospheric oxygen conditions. The protocol will work in both cases, however, the formation of brown precipitate (iron oxide) can be reduced or eliminated when working under $\mathrm{N}_{2}$ atmosphere and therefore, the stability of the uncoated beads can be increased.

1. Mix $2 \mathrm{~L}$ of $99 \%$ ethanol with $50 \mathrm{ml}$ of $25 \%$ ammonia solution and $\sim 22.5 \mathrm{~g}$ (wet mass) of the synthesized iron oxide MNPs (A) in a heat resistant $2.5 \mathrm{~L}$ bottle using a magnetic stirrer (300$400 \mathrm{rpm}$ ). Switch on the heating and allow the solution to heat up to $\sim 80^{\circ} \mathrm{C}$.

Note: It is very important that the core particles are efficiently distributed in the solution. Otherwise, large clusters of MNPs are coated with silica, leading to a decreased performance of the beads in nucleic acid capture applications.

2. Add $45 \mathrm{ml}$ tetraethyl orthosilicate (TEOS) with constant stirring and incubate for another $30 \mathrm{~min}$. Note: The size of the particles can be controlled using different ratios of core particles and TEOS. The standard ratio used in the above protocol is: $1 \mathrm{~g}$ of paramagnetic core particles to $2 \mathrm{ml}$ TEOS, which results in particles with an average size of $\sim 400 \mathrm{~nm}$. Generally, more TEOS yields larger beads.

3. Add $400 \mathrm{ml}$ of $\mathrm{ddH}_{2} \mathrm{O}$ to the solution.

4. Allow the reaction to proceed for $>4 \mathrm{~h}$ (ideally overnight).

Note: TEOS hydrolyzes spontaneously in water which will result in white silica precipitates which do not contain a paramagnetic core. If large amounts of this precipitate forms, use fresh TEOS for the reaction.

5. Cool the solution to RT. 
6. Separate the coated MNPs using a strong neodymium magnet.

Note: Uncoated MNPs get slowly oxidized over time which is indicated by a brown color of the supernatant after magnetic separation.

7. Wash twice with pure water.

8. Wash twice with pure ethanol.

9. Wash with pure water until the $\mathrm{pH}$ of the solution becomes neutral (3-4 times).

Note: To measure the synthesis yield, magnetically pellet the silica beads, remove the water and weigh the product wet mass. The coated beads can be stored at RT for at least 1 year.

C. BOMB carboxyl-coating

Note: All procedures can be performed under inert $\mathrm{N}_{2}$ atmosphere or atmospheric oxygen conditions. The protocol will work in both cases, however, the formation of brown precipitate (iron oxide) can be reduced or eliminated when working under $\mathrm{N}_{2}$ atmosphere and therefore, the stability of the uncoated beads can be increased.

1. Mix $1 \mathrm{~g}$ (wet mass) of the synthesized iron oxide MNPs (A) dispersed in $45 \mathrm{ml}$ of water and 1.15 g sodium dodecyl sulfate with $200 \mathrm{ml}$ of purified, degassed water in a round bottom flask using a magnetic stirrer (300-400 rpm). Switch on the heating and allow the solution to heat up to $\sim 70{ }^{\circ} \mathrm{C}$.

2. Add $0.96 \mathrm{ml}$ of methacrylic acid (MAA) into the flask.

Note: The $\mathrm{pH}$ drops to about 3.

3. Equilibrate the reaction mixture for about $45 \mathrm{~min}$ while keeping the temperature.

4. Add $1.98 \mathrm{~g}$ of the initiator potassium persulfate to the solution.

5. Let the polymerization reaction progress at $\sim 70{ }^{\circ} \mathrm{C}$ for $2 \mathrm{~h}$.

6. Cool the solution to RT.

7. Separate the coated MNPs from the free MAA and PMAA by concentrating the MNPs with a strong neodymium magnet and discarding the supernatant.

Note: Uncoated MNPs get slowly oxidized over time which is indicated by a brown color of the supernatant after magnetic separation.

8. Disperse the isolated paramagnetic nanoparticles in deionized water (e.g., in an ultrasonic bath), followed by magnetic extraction as described in Step C7.

9. Wash the beads with $\mathrm{ddH}_{2} \mathrm{O}$ at least 5 times or until the detergent is completely removed and disperse them in $250 \mathrm{ml}$ of $\mathrm{ddH}_{2} \mathrm{O}$.

Note: To measure the synthesis yield, magnetically pellet the silica beads, remove the water and weigh the product wet mass. The coated beads can be stored at RT for at least 1 year.

D. Benchmarking the BOMB paramagnetic beads

The success of the core particle synthesis, as well as the coating reactions, can be monitored by electron microscopy (TEM, SEM), as well as to a limited extent with light microscopy (LM). The resulting microscopy pictures are displayed in Figures 1C, 1D and 1E. A successful synthesis should 
yield predominantly single spherical beads which display paramagnetic properties and cluster when exposed to a strong magnetic field. It is also possible to assess further properties of the beads using X-ray Powder Diffraction (XRD), if this information is needed for the respective application. However, for standard applications such as nucleic acid extraction this is not necessary.

We also recommend benchmarking the performance of the beads from each synthesis, and testing them if stored for a longer time. For this, we suggest titring the amount of beads and/or binding buffer in a clean-up of a DNA standard, as described in detail in BOMB protocol \#4.1 (https://bomb.bio/protocols). Furthermore, we recommend comparing the results for each application to available commercial setups. A protocol on how to do this can also be found in BOMB protocol \#4.1.

E. Nucleic acid purification with the BOMB paramagnetic beads

The synthesized beads have been tested extensively for a great variety of previously published protocols (Oberacker et al., 2019). Here we display exemplary results for the extraction of nucleic acids from E. coli (Figure 2A), HEK293 cells (Figure 2B) and various mammalian tissues (Figure 2C) using the silica-beads described above. All protocols are also publicly available on the BOMB.bio homepage (https://bomb.bio/protocols).

\section{Data analysis}

All data underlying the results shown above can be found in the supporting information of the original paper (Oberacker et al., 2019) in PLOS Biology.

\section{Recipes}

1. $2 \mathrm{M} \mathrm{NaOH}$

Dissolve in water

Can be stored at RT for at least 12 months

2. $0.1 \mathrm{M} \mathrm{HCl}$

Dilute in water from stock solution

Can be stored at RT for at least 12 months

3. Fe2/3 solution (freshly prepared)

$3.24 \mathrm{~g} \mathrm{FeCl}_{3}(0.333 \mathrm{M})$

$2.00 \mathrm{~g} \mathrm{FeCl}_{2} \cdot 4 \mathrm{H}_{2} \mathrm{O}(0.167 \mathrm{M})$

Add $60 \mathrm{ml} 0.1 \mathrm{M} \mathrm{HCl}$ and dissolve by magnetic stirring, sterile filter with a $0.2 \mu \mathrm{m}$ syringe filter using a $60 \mathrm{ml}$ syringe 


\section{Acknowledgments}

The authors would like to thank all contributors of the initial publication for their contributions to the BOMB project and the development of protocols employing paramagnetic beads for nucleic acid purifications. We would also like to thank the wider research community for offering unpublished information and resources concerning paramagnetic bead preparation and utility.

Author's contributions: The idea was conceived by TPJ and TH. Synthesis protocol setup and optimization were done by TPJ. Nucleic acid extraction data as well as light microscopy analysis were provided by PS, DB and PO. The electron microscope analysis was done by $\mathrm{KH}$. The manuscript draft was written by PO, TPJ and TH. All authors contributed to the editing of the manuscript and approved its final version.

\section{Competing interests}

The authors declare no conflict of interest.

\section{References}

1. Aggarwal, A., Saxena, R., Wang, B. and Caneba, G. T. (1996). Studies of the polymerization of methacrylic acid via free-radical retrograde precipitation polymerization process. $J$ Appl Polym Sci 62(12): 2039-2051.

2. Choi, J., Kim, J. C., Lee, Y. B., Kim, I. S., Park, Y. K. and Hur, N. H. (2007). Fabrication of silicacoated magnetic nanoparticles with highly photoluminescent lanthanide probes. Chem Commun (Camb)(16): 1644-1646.

3. DeAngelis, M. M., Wang, D. G. and Hawkins, T. L. (1995). Solid-phase reversible immobilization for the isolation of PCR products. Nucleic Acids Res 23(22): 4742-4743.

4. Hawkins, T. L., O'Connor-Morin, T., Roy, A. and Santillan, C. (1994). DNA purification and isolation using a solid-phase. Nucleic Acids Res 22(21): 4543-4544.

5. Houshiar, M., Zebhi, F., Jafari, Z., Alidoust, A. and Askari, Z. (2014). Synthesis of cobalt ferrite $\left(\mathrm{CoFe}_{2} \mathrm{O}_{4}\right)$ nanoparticles using combustion, coprecipitation, and precipitation methods: A comparison study of size, structural, and magnetic properties. J Magn Magn Mater 371: 43-48

6. Kim, J. W., Kim, L. U. and Kim, C. K. (2007). Size control of silica nanoparticles and their surface treatment for fabrication of dental nanocomposites. Biomacromolecules 8(1): 215-222.

7. Majidi, S., Sehrig, F. Z., Farkhani, S. M., Goloujeh, M. S. and Akbarzadeh, A. (2016). Current methods for synthesis of magnetic nanoparticles. Artif Cells Nanomed Biotechnol 44(2): 722734.

8. Oberacker, P., Stepper, P., Bond, D. M., Hohn, S., Focken, J., Meyer, V., Schelle, L., Sugrue, V. J., Jeunen, G. J., Moser, T., Hore, S. R., von Meyenn, F., Hipp, K., Hore, T. A. and Jurkowski, 
T. P. (2019). Bio-On-Magnetic-Beads (BOMB): Open platform for high-throughput nucleic acid extraction and manipulation. PLoS Biol 17(1): e3000107.

9. Rohland, N. and Reich, D. (2012). Cost-effective, high-throughput DNA sequencing libraries for multiplexed target capture. Genome Res 22(5): 939-946.

10. Singh, D., McMillan, J. M., Liu, X. M., Vishwasrao, H. M., Kabanov, A. V., Sokolsky-Papkov, M. and Gendelman, H. E. (2014). Formulation design facilitates magnetic nanoparticle delivery to diseased cells and tissues. Nanomedicine (Lond) 9(3): 469-485.

11. Stöber, W., Fink, A. and Bohn, E. (1968). Controlled growth of monodisperse silica spheres in the micron size range. $J$ Colloid Interf Sci 26(1): 62-69.

12. Wu, W., Wu, Z., Yu, T., Jiang, C. and Kim, W. S. (2015). Recent progress on magnetic iron oxide nanoparticles: synthesis, surface functional strategies and biomedical applications. Sci Technol Adv Mater 16(2): 023501. 